\title{
Analytical studies assessing the association between extreme precipitation or temperature and drinking water-related waterborne infections: a review
}

Bernardo R Guzman Herrador ${ }^{1 *}$, Birgitte Freiesleben de Blasio ${ }^{1,2}$, Emily MacDonald ${ }^{1,3}$, Gordon Nichols ${ }^{4,5,6,7}$, Bertrand Sudre ${ }^{4}$, Line Vold ${ }^{1}$, Jan C Semenza ${ }^{4}$ and Karin Nygård ${ }^{1}$

\begin{abstract}
Determining the role of weather in waterborne infections is a priority public health research issue as climate change is predicted to increase the frequency of extreme precipitation and temperature events. To document the current knowledge on this topic, we performed a literature review of analytical research studies that have combined epidemiological and meteorological data in order to analyze associations between extreme precipitation or temperature and waterborne disease.

A search of the databases Ovid MEDLINE, EMBASE, SCOPUS and Web of Science was conducted, using search terms related to waterborne infections and precipitation or temperature. Results were limited to studies published in English between January 2001 and December 2013.

Twenty-four articles were included in this review, predominantly from Asia and North-America. Four articles used waterborne outbreaks as study units, while the remaining articles used number of cases of waterborne infections. Results presented in the different articles were heterogeneous. Although most of the studies identified a positive association between increased precipitation or temperature and infection, there were several in which this association was not evidenced. A number of articles also identified an association between decreased precipitation and infections. This highlights the complex relationship between precipitation or temperature driven transmission and waterborne disease. We encourage researchers to conduct studies examining potential effect modifiers, such as the specific type of microorganism, geographical region, season, type of water supply, water source or water treatment, in order to assess how they modulate the relationship between heavy rain events or temperature and waterborne disease. Addressing these gaps is of primary importance in order to identify the areas where action is needed to minimize negative impact of climate change on health in the future.
\end{abstract}

Keywords: Review, Precipitation, Rainfall, Temperature, Waterborne infection

\section{Background}

Mechanisms through which extreme precipitation, both increased and decreased, can contribute to the occurrence of waterborne infections are well documented. Heavy precipitation events increase the likelihood of water supply contamination due to the risk of sewer overflows [1]. Aging water treatment and distribution systems are

\footnotetext{
* Correspondence: BernardoRafael.Guzman.Herrador@fhi.no

'Department of Infectious Disease Epidemiology, Norwegian Institute of

Public Health, Oslo, Norway

Full list of author information is available at the end of the article
}

particularly susceptible to heavy precipitation events, increasing the vulnerability of the drinking water supply. On the other hand, low precipitation may contribute to waterborne infections by increasing the percentage of sewage effluent in rivers when rainfall decreases or by increasing risk of groundwater contamination when the water table drops. In addition, many infectious agents and their vector and reservoir cycles are sensitive to temperature conditions [2].

A considerable amount of research is being conducted to map and assess risks, vulnerabilities and the impact of 
climate change in waterborne disease [3-5]. A recently published review [6] identified waterborne outbreaks potentially linked to an extreme water-related weather event and assessed how the different types of extreme weather events impact the occurrence of waterborne disease. Authors concluded that improving the understanding of the effects that different extreme water-related weather events have on waterborne disease is an important step towards finding ways to mitigate the risks.

Both the World Health Organization (WHO) and the European Centre for Disease Prevention and Control (ECDC) have emphasized the need for strengthening partnerships between health and climate experts, to improve scientific evidence of the linkages between health and climate drivers $[7,8]$. Despite the abundance of meteorological and epidemiological registries and databases, these are often not linked, preventing a more comprehensive understanding of potential associations [8]. Other publications have also highlighted additional obstacles to data access for research related to climate and water [9], and claim a reprioritization of public health research to ensure that funding is dedicated to explicitly studying the effects of changes in climate variables on food- and waterborne diseases [10].

To document the available knowledge, we performed a literature review of analytical research studies that have combined epidemiological and meteorological data to assess associations between extreme precipitation or air temperature and waterborne infections. This will help to identify specific areas where more specific research on this topic is needed.

\section{Methods}

\section{Search strategy}

The keywords used for searching relevant articles included both general and specific terms related to water, waterborne infections and precipitation or temperature related conditions (Table 1). These three groups of keywords were combined. The search strategy was run in the medical databases Ovid MEDLINE and EMBASE and in the multidisciplinary databases SCOPUS and Web of Science. Titles and abstracts of publications were searched for keywords. In order to focus on the most relevant and recent research, the search was limited to studies involving humans published in English between January 2001 and
December 2013. In addition, a snowballing technique was used to review the reference lists of selected studies to identify additional articles.

\section{Data extraction strategy}

Two independent reviewers screened titles for relevance obtained after running the search strategy. In a second step, selected abstracts were screened using the inclusion and exclusion criteria specified in Table 2 . The full text of relevant studies were retrieved and assessed for eligibility. A sample of ten articles was reviewed by two independent reviewers in order to determine what data should be extracted. Dummy tables were designed for this purpose.

The following data were extracted from the articles and included in Tables 3 and 4: first author, publication year, location of study (continent, country or region), study period (in years), waterborne infection studied and data source, study objective, exposure variable studied (precipitation or/and temperature) and data source, analytical methods used, additional information (whether the study took into account in the analysis seasonality, water source, water treatment, or water supply involved), and main associations and conclusions found in the study. Articles were classified according to the study units used (outbreaks or cases of infection).

\section{Results}

Once duplicates were removed, a total of 1907 titles were obtained using the initial search terms. Following screening of titles, results were limited to 457 articles. After screening abstracts for relevance, 79 full-text articles were read full text, of which 57 were excluded. Two articles were included after checking the reference lists of the already selected articles. In total, 24 analytical research articles, in which the association between extreme precipitation or air temperature and waterborne infections had been assessed, were included in the literature review (Figure 1).

\section{Studies of drinking water-related waterborne infections, geographical location and data sources \\ Articles using outbreaks as study units $(n=4)$}

Four studies used drinking water related waterborne outbreaks as study units [11-14]. Two articles presented

Table 1 Keywords used for searching in the literature

\begin{tabular}{ll}
\hline Thematic areas & Specific terms* \\
\hline Water source & Water, water supply, groundwater, surface water, water purification, water disinfection, sewage \\
Waterborne infection & Waterborne, gastroenteritis, outbreak, campylobacteriosis, Escherichia coli, cholera, cryptosporiosis, \\
Weather conditions & Climate, weather, precipitation, rain, rainfall, temperature, humidity, season, flood, drought, snow
\end{tabular}

"Terms in the same box were combined with "or" in the search. Terms in the different rows were combined with "and" in the search. 
Table 2 Inclusion and exclusion criteria

\begin{tabular}{ll}
\hline Inclusion criteria & Analytical research studies in which the main objective was \\
& To estimate the association between extreme precipitation or temperature and drinking \\
& water-related waterborne outbreaks or infections \\
& Study type: \\
& -Outbreak reports reporting a single outbreak event. \\
& -Pure discussion papers or reviews without specific statistical analysis and results presented. \\
& -Studies without statistical analysis of associations (i.e. surveys). \\
& Events presented: \\
& -Outbreaks or trends of food-borne and vector-borne outbreaks or infections \\
& -Study of environmental conditions other than precipitation or air temperature \\
& - Main route of transmission other than drinking water. \\
& -Estimation of the association between extreme precipitation or temperature and concentration \\
of microorganisms in water, but without data on human illness presented in the paper. & -Study of seasonality not related to weather or climate data. \\
& Population: Humans \\
Search strategy limited to: & Publication year: January 2001-December 2013 \\
& Language: English
\end{tabular}

studies that were performed using data from North America (Canada and United States) [11,14] while one used data from Europe (England and Wales) [13]. One study included data from several continents [12]. There were different data sources used to obtain outbreak data, including surveillance data, publicly available databases, previous published compilations and unpublished reports. The four studies assessed the association between outbreaks and precipitation. Two of them also studied the relationship with temperature. Meteorological data under study were obtained from records available at international organizations or from readings from the relevant weather stations.

Articles using cases of infection as study units $(n=20)$ The remaining 20 articles used cases of infection as study units [15-34]. Most of the articles $(n=7)$ were performed in Asia (Bangladesh, Indonesia, Vietnam, India, Taiwan and China) [23,24,27,29-31,33]. Four were performed in North America (United States and Canada) $[16,21,25,26]$, four in Oceania (Australia, New Zealand and Pacific Islands) [17,20,28,34], two in Europe (Sweden; and England and Wales) [18,22], one in central America (Haiti) [15], and one in Africa (Lusaka) [19]. One article used data from more than one continent, Asia and Central America [32].

The most common approach was to use cases of gastrointestinal infections without specifying the type of microorganism $(n=6)$. Among those studies focusing on specific microorganisms, cholera was most frequently studied $(n=6)$, followed by campylobacteriosis $(n=3)$ and typhoid fever $(n=3)$. Other infections, such as shigellosis, cryptosporidiosis, giardiasis, hepatitis A and paratyphoid fever, were also studied.

Cases of infection were obtained from several sources, including surveillance data, clinical records and registries, governmental reports and nurse advice telephone lines. All studies assessed the association between cases of infection and precipitation, while eleven of them also examined the relationship with temperature. The meteorological data under study were obtained from records available at international organizations, satellite sensors, gauge estimates, interviews or from local weather stations.

\section{Definition extreme precipitation or temperature, covariates and statistical analysis}

The definition of extreme weather events varied across the studies. There were different ways of categorizing meteorological variables, according to the amount or range of precipitation (i.e. groups including different categories; accumulated; smoothed using a certain number of days moving average; dichotomous, above and below a threshold; total in a given period; exceeded the upper limit of a given reference range). Only seven articles presented analyses stratified by water source or type of water supply, aiming to disentangle differences in the association with the occurrence of waterborne infections.

Analysis using Poisson regression or other types of count model regression was the most commonly adopted method to investigate whether variation in disease occurrence could be partly explained by changes in variables related to extreme weather events. Count model regression was used in eleven studies, one with outbreaks [12] and 
Table 3 Region, study period, waterborne infections and data sources in the included articles by type of study unit

\begin{tabular}{|c|c|c|c|c|c|c|}
\hline $\begin{array}{l}\text { Study } \\
\text { units }\end{array}$ & $\begin{array}{l}\text { First author } \\
\text { publication year }\end{array}$ & Continent & Country/Region & Study period & Waterbone disease under study & Waterborne disease Data source \\
\hline \multirow[t]{4}{*}{ Outbreaks } & Yang [12]; 2012 & Global & - & 1991-2008 (18 years) & $\begin{array}{l}\text { Drinking water related waterborne } \\
\text { disease outbreaks (+ other } \\
\text { water-associated diseases) }\end{array}$ & $\begin{array}{l}\text { Database developed by the Global } \\
\text { Infectious Disease Epidemiology Network } \\
\text { (GIDEON) }\end{array}$ \\
\hline & Curriero [14]; 2001 & North America & United States & 1948-1994 (47 years) & $\begin{array}{l}\text { Drinking water related waterborne } \\
\text { disease outbreaks with contamination } \\
\text { at the water source }\end{array}$ & Surveillance data at national level \\
\hline & Thomas [11]; 2006 & North America & Canada & 1975-2001 (27 years) & $\begin{array}{l}\text { Drinking water related waterborne } \\
\text { disease outbreaks }\end{array}$ & Published compilation at national level \\
\hline & Nichols [13]; 2009 & Europe & England and Wales & 1910-1999 (90 years) & $\begin{array}{l}\text { Drinking water related waterborne } \\
\text { disease outbreaks }\end{array}$ & $\begin{array}{l}\text { Medline search, published papers and } \\
\text { unpublished reports }\end{array}$ \\
\hline \multirow[t]{18}{*}{$\begin{array}{l}\text { Cases of } \\
\text { infection }\end{array}$} & Tornevi [22]; 2013 & Europe & Gothenburg, Sweden & 2007-2011 (5 years) & $\begin{array}{l}\text { Telephone calls to acute } \\
\text { gastrointestinal illnesses }\end{array}$ & Nurse advice line \\
\hline & Louis [18]; 2005 & Europe & England and Wales & 1990-1999 (10 years) & Campylobacteriosis cases & Surveillance data at national level \\
\hline & \multirow[t]{3}{*}{ Eisenberg [15]; 2013} & \multirow[t]{3}{*}{ Central America } & \multirow[t]{3}{*}{ Haiti } & \multirow[t]{3}{*}{ 2010-2011 } & \multirow[t]{3}{*}{ Cholera cases } & Registry at a hospital \\
\hline & & & & & & Internally displaced person camp data \\
\hline & & & & & & Reports at the ministry \\
\hline & White [25]; 2009 & North America & Philadelphia, United States & 1994-2007 (14 years) & Campylobacteriosis cases & Surveillance data at national level \\
\hline & Drayna [26]; 2010 & North America & Wisconsin, United States & 2002-2007 (6 years) & $\begin{array}{l}\text { Physician visits of gastrointestinal } \\
\text { infections/diarrhea }\end{array}$ & Administrative records \\
\hline & Teschke [21]; 2010 & North America & Vancouver, Canada & 1995-2003 (9 years) & $\begin{array}{l}\text { Physician visits and hospitalization } \\
\text { records of various gastrointestinal } \\
\text { diseases with potential to be waterborne }\end{array}$ & Administrative records \\
\hline & Harper [16]; 2011 & North America & Nunatsiavut, Canada & 2005-2008 (4 years) & Gastrointestinal illness related visits & Administrative records \\
\hline & Hashizume [27]; 2007 & Asia & Dhaka, Bangladesh & 1996-2002 (7 years) & $\begin{array}{l}\text { Weekly number of patients visiting a } \\
\text { hospital due to non-cholera diarrhea }\end{array}$ & Administrative records \\
\hline & Vollaard [23]; 2004 & Asia & Jakarta, Indonesia & 2001-2003 (3 years) & Typhoid or paratyphoid fever cases & $\begin{array}{l}\text { Consultations at hospitals and outpatient } \\
\text { health centers }\end{array}$ \\
\hline & Kelly-Hope [33]; 2007 & Asia & Vietnam & 1991-2001 (11 years) & $\begin{array}{l}\text { Shigellosis, cholera and typhoid fever } \\
\text { cases }\end{array}$ & $\begin{array}{l}\text { Surveillance data at national level and } \\
\text { published papers and unpublished reports }\end{array}$ \\
\hline & \multirow[t]{2}{*}{ Emch [31]; 2008} & \multirow[t]{2}{*}{ Asia } & $\begin{array}{l}\text {-Hue and Nha Tranng, } \\
\text { Vietnam }\end{array}$ & $-1985-2003$ (23 years) & \multirow[t]{2}{*}{ Cholera cases } & \multirow[t]{2}{*}{$\begin{array}{l}\text { Records from a research centre/surveillance } \\
\text { data at national level }\end{array}$} \\
\hline & & & -Matlab,Bangladesh & $-1983-2003$ (21 years) & & \\
\hline & \multirow{2}{*}{$\begin{array}{l}\text { Constantin de Magny } \\
\text { [30]; } 2008\end{array}$} & \multirow[t]{2}{*}{ Asia } & -Kolkata, India & \multirow[t]{2}{*}{ 1997-2006(10 years) } & \multirow[t]{2}{*}{ Cholera cases } & Administrative records \\
\hline & & & -Matlab, Bangladesh & & & Records from a research center \\
\hline & Wang [24]; 2012 & Asia & Guizhou, China & 1984-2007 (24 years) & Typhoid and paratyphoid fever cases & Surveillance data at national level \\
\hline & Chen [29]; 2012 & Asia & Taiwan & 1994-2008 (15 years) & $\begin{array}{l}\text { Hepatitis A, enteroviruses, shigellosis } \\
\text { cases }\end{array}$ & Surveillance data at national level \\
\hline
\end{tabular}


Table 3 Region, study period, waterborne infections and data sources in the included articles by type of study unit (Continued)

\begin{tabular}{|c|c|c|c|c|c|}
\hline \multirow[t]{2}{*}{ Jutla,[32]; 2013} & \multirow{2}{*}{$\begin{array}{l}\text { Asia and Central } \\
\text { America }\end{array}$} & -Northern India and Pakistan & $-1875-1900$ (26 years) & \multirow[t]{2}{*}{ Cholera cases } & \multirow{2}{*}{$\begin{array}{l}\text { Reports from the Government and } \\
\text { previous published data }\end{array}$} \\
\hline & & -Haiti & -2010 & & \\
\hline Singh [20]; 2001 & $\begin{array}{l}\text { Oceania and } \\
\text { Australia }\end{array}$ & Pacific Islands & $\begin{array}{l}\text { 1978-1998, with two } \\
\text { missing years( } 19 \text { years) }\end{array}$ & Diarrhea cases & Surveillance data at national level \\
\hline $\mathrm{Hu}[17] ; 2007$ & Oceania and Australia & Brisbane, Australia & 1996-2004 (9 years) & Cryptosporidiosis cases & Surveillance data from the regional level \\
\hline Rind [34]; 2010 & Oceania and Australia & New Zealand & 1997-2005 (9 years) & Campylobacteriosis cases & Surveillance data at national level \\
\hline Britton [28]; 2010 & Oceania and Australia & New Zealand & 1997-2006 (10 years) & Cryptosporidiosis and Giardiasis cases & Surveillance data at national level \\
\hline Sasaki [19]; 2009 & Africa & Lusaka, Zambia & $2003-2004 ; 2005-2006$ & Cholera cases & Records at a treatment centre \\
\hline
\end{tabular}

Literature Review $(n=24)$. 
Table 4 Region, objective, exposure variables and data sources, analytical method, results and conclusions in the included articles by type of study unit

\begin{tabular}{|c|c|c|c|c|c|c|}
\hline $\begin{array}{l}\text { Study } \\
\text { units }\end{array}$ & $\begin{array}{l}\text { First author } \\
\text { publication year }\end{array}$ & Objective & $\begin{array}{l}\text { Exposure variable under study } \\
\text { (Precipitation/Air temperature) }\end{array}$ & $\begin{array}{l}\text { Exposure variable } \\
\text { data source }\end{array}$ & Analytical method & $\begin{array}{l}\text { Additional } \\
\text { information }\end{array}$ \\
\hline \multirow[t]{3}{*}{ Outbreaks } & Yang [12]; 2012 & Risk factors associated & Average precipitation per year & \multirow{2}{*}{$\begin{array}{l}\text { Records from } \\
\text { international } \\
\text { organizations }\end{array}$} & \multirow{2}{*}{$\begin{array}{l}\text { Zero-inflated Poisson } \\
\text { regression }\end{array}$} & \multirow[t]{2}{*}{-} \\
\hline & & $\begin{array}{l}\text { distributions of } \\
\text { water-associated } \\
\text { outbreaks }\end{array}$ & $\begin{array}{l}\text { Global average accumulated } \\
\text { temperature (degree-days) }\end{array}$ & & & \\
\hline & $\begin{array}{l}\text { Curriero [14]; } \\
2001\end{array}$ & $\begin{array}{l}\text { Association between } \\
\text { extreme precipitation } \\
\text { and waterborne disease } \\
\text { outbreaks. }\end{array}$ & $\begin{array}{l}\text { Extreme precipitation above certain } \\
\text { threshold by watershed }\end{array}$ & $\begin{array}{l}\text { Readings of } \\
\text { relevant weather } \\
\text { stations }\end{array}$ & $\begin{array}{l}\text { Monte Carlo version of } \\
\text { the Fisher exact test }\end{array}$ & $\begin{array}{l}\text { Analysis } \\
\text { stratified by } \\
\text { water source } \\
\text { and control for } \\
\text { seasonality }\end{array}$ \\
\hline
\end{tabular}

Association found

Waterborne diseases are inversely

related to average annual

precipitation.

No association between

temperature and waterborne disease.

Positive association between

extreme precipitation and

outbreak occurrence

Both for surface water (strongest association during the month of the outbreak) and groundwater contamination (2-month prior to the outbreaks)

\begin{tabular}{|c|c|c|}
\hline \multirow[t]{2}{*}{$\begin{array}{l}\text { Thomas [11]; } \\
2006\end{array}$} & \multirow[t]{2}{*}{$\begin{array}{l}\text { Test the association } \\
\text { between high impact } \\
\text { weather event and } \\
\text { waterborne disease } \\
\text { outbreaks }\end{array}$} & $\begin{array}{l}\text { Accumulated precipitation, smoothed using } \\
\text { a five-day moving average, maximum } \\
\text { percentile of the accumulated } \\
\text { precipitation amount, number of days } \\
\text { between the maximum percentile and } \\
\text { the case or control onset day temperature }\end{array}$ \\
\hline & & $\begin{array}{l}\text { Degree-days above } 0 \mathrm{C} \text {, the maximum } \\
\text { temperature smoothed using a five-day } \\
\text { moving average, and the number of } \\
\text { days between max temp and the case } \\
\text { and the control onset day }\end{array}$ \\
\hline
\end{tabular}

Nichols [13]; $\quad$ Association between

2009 precipitation and

outbreaks of drinking

Readings of case-crossover analysis seasonality

Positive association between accumulated precipitation percentile and outbreak

occurrence

Positive association between degree-days above $0 \mathrm{C}$ and outbreak occurrence

Cumulative precipitation in four time periods prior to each outbreak

Readings of Time-stratified matched Water source, relevant weather case-crossover analysis season, water stations

Excessive precipitation: total number of days in which the precipitation exceeded a certain upper limit

supply

considered as effect modifiers
Cases of Tornevi [22]. infection 2013

Determine if variation in Daily precipitation

the incidence of acute

gastrointestinal illnesse

is associated with

upstream precipitation

Investigate the
relationship betwee

environmenta

Precipitation divided into three

categories up and down a certain

threshold
Readings of

relevant weather

stations

Poisson regression

(with nonlinear

distributed lag

function)

Readings of

relevant weathe

Time series analysis

stations
Positive association with excess precipitation over the previous week and low precipitation in the three weeks before the week of the outbreak

Greater risk in groundwater, spring and private wate supplies. These interactions were non-significant when including them together in a mode suggesting confounding.

Control for seasonality

Heavy precipitation was associated with increased calls.

Seasonality and Campylobacter rates were water supply correlated with temperature

No association with precipitation 
Table 4 Region, objective, exposure variables and data sources, analytical method, results and conclusions in the included articles by type of study unit

\begin{tabular}{|c|c|c|c|c|c|c|}
\hline & $\begin{array}{l}\text { conditions and } \\
\text { Campylobacter } \\
\text { infections }\end{array}$ & Daily max and minimum temperature & & & $\begin{array}{l}\text { also included in } \\
\text { the study }\end{array}$ & $\begin{array}{l}\text { No association with surface } \\
\text { water. }\end{array}$ \\
\hline \multirow{5}{*}{$\begin{array}{l}\text { Eisenberg [15]; } \\
2013\end{array}$} & \multirow{5}{*}{$\begin{array}{l}\text { Examine the } \\
\text { relationship between } \\
\text { cholera and } \\
\text { precipitation in Haiti } \\
\text { including statistical and } \\
\text { dynamic models }\end{array}$} & \multirow[t]{5}{*}{ Cumulative daily totals for precipitation } & \multirow{5}{*}{$\begin{array}{l}\text { Rain gauges and } \\
\text { satellite } \\
\text { measurements }\end{array}$} & Statistical modeling & \multirow{5}{*}{$\begin{array}{l}\text { Control for } \\
\text { seasonality }\end{array}$} & \multirow{5}{*}{$\begin{array}{l}\text { All analysis support a strong } \\
\text { positive association between } \\
\text { precipitation and cholera } \\
\text { incidence in Haiti }\end{array}$} \\
\hline & & & & $\begin{array}{l}\text { Quasi-Poisson } \\
\text { regression (with } \\
\text { nonlinear distributed } \\
\text { lag function) }\end{array}$ & & \\
\hline & & & & $\begin{array}{l}\text { Granger Causality Wald } \\
\text { Test }\end{array}$ & & \\
\hline & & & & Case-crossover analysis & & \\
\hline & & & & Dynamic modeling & & \\
\hline \multirow[t]{3}{*}{ White [25]; 2009} & \multirow{3}{*}{$\begin{array}{l}\text { Association between } \\
\text { environmental factors } \\
\text { and campylobacter } \\
\text { infection }\end{array}$} & Precipitation & \multirow{3}{*}{$\begin{array}{l}\text { Readings of } \\
\text { relevant weather } \\
\text { stations }\end{array}$} & Poisson regression & \multirow{3}{*}{$\begin{array}{l}\text { Control for } \\
\text { seasonality }\end{array}$} & \multirow{3}{*}{$\begin{array}{l}\text { Weekly incidence was associated } \\
\text { with increasing mean } \\
\text { temperature. } \\
\text { No association with precipitation }\end{array}$} \\
\hline & & \multirow[t]{2}{*}{ Temperature } & & \multirow{2}{*}{$\begin{array}{l}\text { Time-stratified matched } \\
\text { case-crossover analysis }\end{array}$} & & \\
\hline & & & & & & \\
\hline Drayna [26]; 2010 & $\begin{array}{l}\text { Association between } \\
\text { precipitation and acute } \\
\text { gastrointestinal illness in } \\
\text { pediatric population }\end{array}$ & $\begin{array}{l}\text { Total daily precipitation, extreme } \\
\text { considered above a certain percentile }\end{array}$ & $\begin{array}{l}\text { Readings of } \\
\text { relevant weather } \\
\text { stations }\end{array}$ & $\begin{array}{l}\text { Autoregressive moving } \\
\text { average (ARMA) model }\end{array}$ & $\begin{array}{l}\text { Control for } \\
\text { seasonality }\end{array}$ & $\begin{array}{l}\text { Positive association between } \\
\text { precipitation and daily visits }\end{array}$ \\
\hline \multirow[t]{4}{*}{$\begin{array}{l}\text { Teschke [21]; } \\
2010\end{array}$} & \multirow[t]{4}{*}{$\begin{array}{l}\text { Association between } \\
\text { the incidence of } \\
\text { intestinal infections and } \\
\text { environmental factors }\end{array}$} & \multirow[t]{4}{*}{$\begin{array}{l}\text { Precipitation categories according } \\
\text { accumulated millimeters of rain over } \\
\text { certain periods }\end{array}$} & \multirow[t]{4}{*}{$\begin{array}{l}\text { Readings of } \\
\text { relevant weather } \\
\text { stations }\end{array}$} & \multirow[t]{4}{*}{ Logistic regression } & \multirow{4}{*}{$\begin{array}{l}\text { Season, water } \\
\text { supply, water } \\
\text { source, } \\
\text { disinfection and } \\
\text { well depth } \\
\text { included as } \\
\text { variables }\end{array}$} & $\begin{array}{l}\text { The association between } \\
\text { incidence of disease and } \\
\text { precipitation did not remain } \\
\text { when controlling for other } \\
\text { variables }\end{array}$ \\
\hline & & & & & & $\begin{array}{l}\text { Water chlorination was } \\
\text { associated with reduced } \\
\text { physician visits }\end{array}$ \\
\hline & & & & & & $\begin{array}{l}\text { Two water systems with the } \\
\text { highest proportion of surface } \\
\text { water had increased incidence }\end{array}$ \\
\hline & & & & & & $\begin{array}{l}\text { Private well water and well } \\
\text { depth were not associated with } \\
\text { increased risk }\end{array}$ \\
\hline \multirow[t]{3}{*}{ Harper; [16]; 2011} & \multirow{3}{*}{$\begin{array}{l}\text { Association between } \\
\text { weather variables and } \\
\text { gastrointestinal-related } \\
\text { clinic visits }\end{array}$} & Total daily precipitation & \multirow{3}{*}{$\begin{array}{l}\text { Readings of } \\
\text { relevant weather } \\
\text { stations }\end{array}$} & \multirow{3}{*}{$\begin{array}{l}\text { Zero-inflated Poisson } \\
\text { regression }\end{array}$} & \multirow{3}{*}{$\begin{array}{l}\text { Control for } \\
\text { seasonality }\end{array}$} & \multirow{2}{*}{$\begin{array}{l}\text { Positive associations were } \\
\text { observed between high levels of } \\
\text { water volume input } \\
\text { (precipitation + snowmelt) and } \\
\text { IGl clinic visits. }\end{array}$} \\
\hline & & Daily average temperature & & & & \\
\hline & & & & & & No association with temperature \\
\hline $\begin{array}{l}\text { Hashizume [27]; } \\
2007\end{array}$ & $\begin{array}{l}\text { Impact of precipitation } \\
\text { and temperature on the }\end{array}$ & $\begin{array}{l}\text { Daily Precipitation, weekly means } \\
\text { Above/below certain threshold }\end{array}$ & $\begin{array}{l}\text { Records from } \\
\text { national level }\end{array}$ & Poisson regression & $\begin{array}{l}\text { Control for } \\
\text { seasonality }\end{array}$ & $\begin{array}{l}\text { Non-cholera diarrhea cases } \\
\text { increased both above and below }\end{array}$ \\
\hline
\end{tabular}


Table 4 Region, objective, exposure variables and data sources, analytical method, results and conclusions in the included articles by type of study unit

\begin{tabular}{|c|c|c|c|c|c|c|}
\hline & $\begin{array}{l}\text { number of non-cholera } \\
\text { diarrhea cases }\end{array}$ & $\begin{array}{l}\text { Daily minimum/maximum temperature, } \\
\text { weekly means }\end{array}$ & & & & $\begin{array}{l}\text { a threshold level with high and } \\
\text { low precipitation in the } \\
\text { preceding weeks. Cases also } \\
\text { increased with higher } \\
\text { temperature. }\end{array}$ \\
\hline \multirow{2}{*}{$\begin{array}{l}\text { Vollaard [23]; } \\
2004\end{array}$} & \multirow{2}{*}{$\begin{array}{l}\text { Determine risk factors } \\
\text { for typhoid and } \\
\text { paratyphoid fever in an } \\
\text { endemic area }\end{array}$} & Precipitation & \multirow{2}{*}{$\begin{array}{l}\text { Interviews with the } \\
\text { participants }\end{array}$} & \multirow[t]{2}{*}{ Logistic regression } & \multirow[t]{2}{*}{ - } & \multirow{2}{*}{$\begin{array}{l}\text { Flooding was associated with the } \\
\text { occurrence of paratyphoid fever. } \\
\text { Flooding was not associated with } \\
\text { typhoid fever. }\end{array}$} \\
\hline & & $\begin{array}{l}\text { Flooding: defined as inundation of the } \\
\text { house of a participant in the } 12 \text { months } \\
\text { preceding the investigation }\end{array}$ & & & & \\
\hline \multirow{4}{*}{$\begin{array}{l}\text { Kelly-Hope [33]; } \\
2007\end{array}$} & \multirow{4}{*}{$\begin{array}{l}\text { Environmental risk } \\
\text { factors of cholera, } \\
\text { shigellosis and typhoid } \\
\text { fever infections }\end{array}$} & Precipitation & \multirow{4}{*}{$\begin{array}{l}\text { Worldwide maps } \\
\text { generated by the } \\
\text { interpolation of } \\
\text { information from } \\
\text { ground-based } \\
\text { weather stations }\end{array}$} & \multirow[t]{4}{*}{ Linear regression } & \multirow{4}{*}{$\begin{array}{l}\text { Type of water } \\
\text { supply }\end{array}$} & \multirow{2}{*}{$\begin{array}{l}\text { Shigellosis and cholera were } \\
\text { positively associated with } \\
\text { precipitation }\end{array}$} \\
\hline & & \multirow[t]{3}{*}{ Temperature } & & & & \\
\hline & & & & & & $\begin{array}{l}\text { Typhoid fever was not associated } \\
\text { with precipitation }\end{array}$ \\
\hline & & & & & & No association with temperature \\
\hline \multirow[t]{2}{*}{ Emch [31]; 2008} & \multirow{2}{*}{$\begin{array}{l}\text { Association between } \\
\text { cholera and the local } \\
\text { environment }\end{array}$} & Monthly precipitation & \multirow{2}{*}{$\begin{array}{l}\text { Readings of } \\
\text { relevant weather } \\
\text { stations }\end{array}$} & \multirow{2}{*}{$\begin{array}{l}\text { Ordered probit model } \\
\text { to analyze ordinal } \\
\text { outcome (Bangladesh). } \\
\text { Probit model for } \\
\text { dichotomous outcome. } \\
\text { (Vietnam). }\end{array}$} & \multirow[t]{2}{*}{ - } & \multirow{2}{*}{$\begin{array}{l}\text { Temperature and precipitation } \\
\text { not associated with cholera }\end{array}$} \\
\hline & & Monthly temperature & & & & \\
\hline \multirow[t]{2}{*}{$\begin{array}{l}\text { Constantin de } \\
\text { Magny [30]; } 2008\end{array}$} & \multirow{2}{*}{$\begin{array}{l}\text { Association of } \\
\text { environmental } \\
\text { signatures with cholera } \\
\text { epidemics }\end{array}$} & Monthly precipitation & \multirow[t]{2}{*}{$\begin{array}{l}\text { Merged satellite/ } \\
\text { gauge estimates }\end{array}$} & \multirow[t]{2}{*}{$\begin{array}{l}\text { Quasi Poisson } \\
\text { regression }\end{array}$} & \multirow[t]{2}{*}{$\begin{array}{l}\text { Control for } \\
\text { seasonality }\end{array}$} & $\begin{array}{l}\text { Positive association between } \\
\text { cholera and increased } \\
\text { precipitation in Kolkata. }\end{array}$ \\
\hline & & & & & & $\begin{array}{l}\text { No association cholera and } \\
\text { increased precipitation in Matlab }\end{array}$ \\
\hline \multirow[t]{2}{*}{ Wang [24]; 2012} & \multirow[t]{2}{*}{$\begin{array}{l}\text { Impact of meteorological } \\
\text { variations on para/ } \\
\text { typhoid fever (PTF) }\end{array}$} & Monthly cumulative precipitation & \multirow[t]{2}{*}{$\begin{array}{l}\text { Records from } \\
\text { national level }\end{array}$} & $\begin{array}{l}\text {-Spearman's rank } \\
\text { correlation analysis to } \\
\text { analyze the association } \\
\text { between the infection } \\
\text { incidence and the } \\
\text { weather variables }\end{array}$ & \multirow[t]{2}{*}{-} & \multirow[t]{2}{*}{$\begin{array}{l}\text { Temperature and precipitation } \\
\text { were positively associated with } \\
\text { the monthly incidence of PTF }\end{array}$} \\
\hline & & Monthly average temperature & & $\begin{array}{l}\text { Wavelet analysis and } \\
\text { wavelet coherence to } \\
\text { detect the variation of } \\
\text { periodicity over time }\end{array}$ & & \\
\hline Chen [29]; 2012 & $\begin{array}{l}\text { Association between } \\
\text { precipitation and } \\
\text { distribution patterns of } \\
\text { various infectious } \\
\text { diseases, including } \\
\text { water-borne }\end{array}$ & $\begin{array}{l}\text { Precipitation coded as: regular, torrential } \\
\text { and extreme torrential }\end{array}$ & $\begin{array}{l}\text { Readings of } \\
\text { relevant weather } \\
\text { stations }\end{array}$ & $\begin{array}{l}\text { Poisson regression } \\
\text { (with GAM and GAMM) }\end{array}$ & $\begin{array}{l}\text { Control for } \\
\text { seasonality } \\
\text { using monthly } \\
\text { indicator }\end{array}$ & $\begin{array}{l}\text { Daily extreme precipitation levels } \\
\text { correlated with the infections }\end{array}$ \\
\hline Jutla, [32]; 2013 & $\begin{array}{l}\text { Seek an understanding } \\
\text { between hydro- }\end{array}$ & & $\begin{array}{l}\text { Reports from the } \\
\text { government }\end{array}$ & $\begin{array}{l}\text { Spearman's rank } \\
\text { correlation analysis }\end{array}$ & - & $\begin{array}{l}\text { India. -Odds of cholera occurring } \\
\text { were significantly higher when }\end{array}$ \\
\hline
\end{tabular}


Table 4 Region, objective, exposure variables and data sources, analytical method, results and conclusions in the included articles by type of study unit (Continued)

\begin{tabular}{|c|c|c|c|c|c|c|}
\hline & \multirow[t]{3}{*}{$\begin{array}{l}\text { climatological processes } \\
\text { and cholera in } \\
\text { epidemic regions }\end{array}$} & \multirow{3}{*}{$\begin{array}{l}\text { Precipitation and temperature above/ } \\
\text { below average during the previous } \\
\text { months } \\
\text { Daily precipitation and temperature }\end{array}$} & \multirow[t]{3}{*}{ satellite sensors } & & & \multirow{2}{*}{$\begin{array}{l}\text { the temperature was above } \\
\text { climatological average over the } \\
\text { previous two months. Odds of } \\
\text { cholera outbreak was higher } \\
\text { when above average } \\
\text { precipitation occurs. }\end{array}$} \\
\hline & & & & & & \\
\hline & & & & & & $\begin{array}{l}\text { Haiti: Strong correlation between } \\
\text { precipitation and cholera cases. }\end{array}$ \\
\hline \multirow[t]{2}{*}{ Singh [20]; 2001} & \multirow[t]{2}{*}{$\begin{array}{l}\text { Association between } \\
\text { climate variability and } \\
\text { incidence of diarrhea }\end{array}$} & $\begin{array}{l}\text { Precipitation : dichotomous variable } \\
\text { above/below certain threshold }\end{array}$ & \multirow[t]{2}{*}{$\begin{array}{l}\text { Gridded data from } \\
\text { international } \\
\text { institute }\end{array}$} & $\begin{array}{l}\text { Linear regression } \\
\text { Poisson }\end{array}$ & $\begin{array}{l}\text { Control for } \\
\text { seasonality }\end{array}$ & $\begin{array}{l}\text { Positive association between } \\
\text { annual average temperature and } \\
\text { rates of diarrhea }\end{array}$ \\
\hline & & Annual average temperature & & regression & & $\begin{array}{l}\text { Extremes of precipitation were } \\
\text { independently associated with } \\
\text { increased reports of diarrhea }\end{array}$ \\
\hline \multirow[t]{3}{*}{ Hu [17]; 2007} & \multirow{2}{*}{$\begin{array}{l}\text { Impact of weather } \\
\text { variability on the } \\
\text { transmission of } \\
\text { cryptosporidiosis. }\end{array}$} & \multirow[t]{2}{*}{ Monthly total precipitation } & \multirow{3}{*}{$\begin{array}{l}\text { Records from } \\
\text { national level }\end{array}$} & Poisson regression & Control for & Association between \\
\hline & & & & $\begin{array}{l}\text { Seasonal auto- } \\
\text { regression integrated } \\
\text { moving average }\end{array}$ & seasonalıty & $\begin{array}{l}\text { cryptosporialosis and monthly } \\
\text { maximum. temperature }\end{array}$ \\
\hline & $\begin{array}{l}\text { Explore the difference } \\
\text { in the predictive ability } \\
\text { between Poisson } \\
\text { regression and SARIMA } \\
\text { models }\end{array}$ & $\begin{array}{l}\text { Monthly mean minimum/maximum } \\
\text { temperature }\end{array}$ & & (SARIMA) & & \\
\hline \multirow[t]{2}{*}{ Rind [34]; 2010} & \multirow{2}{*}{$\begin{array}{l}\text { Association between } \\
\text { climate factors and } \\
\text { local differences in } \\
\text { campylobacteriosis rates }\end{array}$} & $\begin{array}{l}\text { Monthly mean maximum total } \\
\text { precipitation }\end{array}$ & \multirow[t]{2}{*}{$\begin{array}{l}\text { Records from } \\
\text { research center }\end{array}$} & \multirow[t]{2}{*}{ Linear regression } & \multirow[t]{2}{*}{$\begin{array}{l}\text { Water supply, } \\
\text { seasonality }\end{array}$} & \multirow{2}{*}{$\begin{array}{l}\text { No association found between } \\
\text { temperature and precipitation } \\
\text { and campylobacteriosis rates }\end{array}$} \\
\hline & & $\begin{array}{l}\text { Monthly mean maximum daily } \\
\text { temperatures }\end{array}$ & & & & \\
\hline \multirow[t]{2}{*}{ Britton [28]; 2010} & \multirow{2}{*}{$\begin{array}{l}\text { Association between } \\
\text { precipitation and } \\
\text { ambient temperature } \\
\text { and notifications of } \\
\text { cryptosporidiosis and } \\
\text { giardiasis }\end{array}$} & $\begin{array}{l}\text { Average annual precipitation to } \\
\text { evaporation ratio }\end{array}$ & \multirow{2}{*}{$\begin{array}{l}\text { Mathematical } \\
\text { surfaces fitted to } \\
\text { long run average } \\
\text { climate station } \\
\text { data }\end{array}$} & \multirow[t]{2}{*}{$\begin{array}{l}\text { Negative binomial } \\
\text { regression }\end{array}$} & \multirow[t]{2}{*}{ Water supply } & $\begin{array}{l}\text { Giardiasis: positive association } \\
\text { between precipitation and } \\
\text { temperature. }\end{array}$ \\
\hline & & Average annual temperature & & & & $\begin{array}{l}\text { Cryptosporidiosis: positive } \\
\text { association with precipitation } \\
\text { and negative association with } \\
\text { temperature. The effect of } \\
\text { precipitation was modified by } \\
\text { the quality of the domestic water } \\
\text { supply }\end{array}$ \\
\hline Sasaki [19]; 2009 & $\begin{array}{l}\text { Association between } \\
\text { precipitation patterns } \\
\text { and cholera outbreaks. }\end{array}$ & Daily precipitation data & $\begin{array}{l}\text { Records from } \\
\text { national level and } \\
\text { readings of } \\
\text { relevant weather } \\
\text { stations }\end{array}$ & $\begin{array}{l}\text { Spearman rank } \\
\text { correlation analysis }\end{array}$ & & $\begin{array}{l}\text { Increased precipitation was } \\
\text { associated with the occurrence } \\
\text { of cholera outbreaks }\end{array}$ \\
\hline
\end{tabular}




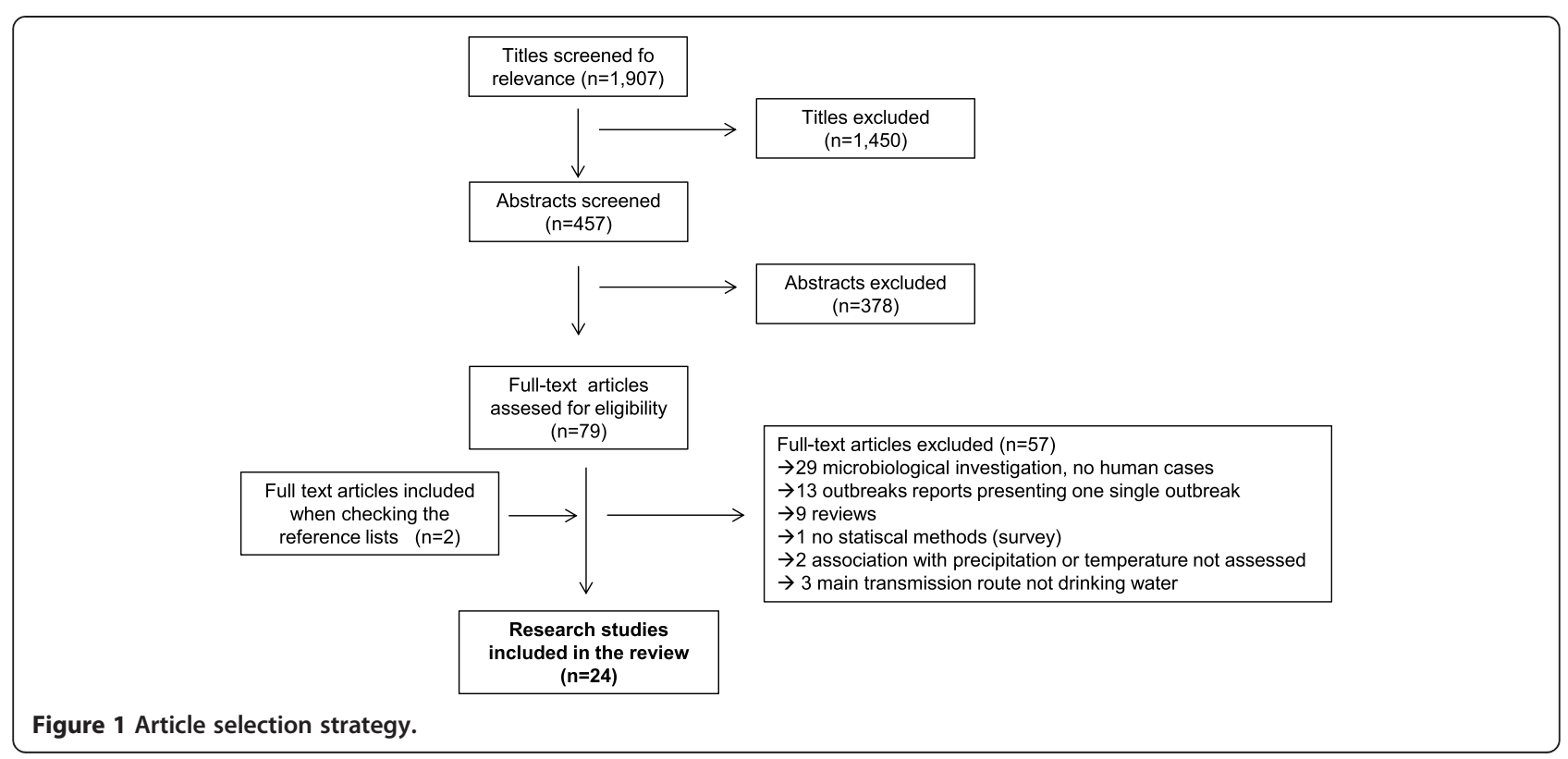

ten with cases of infections [15-17,20,22,25,27-30]. In some cases, the Poisson regression model was adjusted to account for: a) overdispersion, either by estimating an additional dispersion parameter using quasi-Poisson regression models $[15,30]$ or more formally by using negative binomial regression models [28], b) excess zero counts in the observations, by using Zero-inflated Poisson regression models $[12,16]$. Time series data are prone to be influenced by seasonal and long-term variations, which may mask the short-term association between disease and extreme weather events. Seasonal trend decomposition was conducted in different ways, such as by adding trend and seasonal components into the Poisson regression [17], or by using Fourier terms $[20,25,27]$. In some studies, temporal correlations were handled by using generalized additive models (GAM) with time and sometimes other variables related to weather were added as smoother variables [16,29]. Delayed effects and a time varying relationship between the exposure and outcome variables were considered using generalized additive mixed models (GAMM) [29] or nonlinear distributed lag functions [15,22]. Case-crossover analysis was most frequently used when the study units were outbreaks $[11,13]$. It was also used in two studies using cases of infections [15,25]. In this analysis, the weather exposure at the location of an outbreak was compared with the exposures at the same location and same time of the year during control periods without an outbreak through use of conditional logistic regression. The method controls for time-invariant seasonal and geographic differences by design, although it assumes that neither exposure nor confounders change in a systematic way over the course of the study.

\section{Findings of the studies}

All four publications studying outbreaks found an association between precipitation and waterborne disease. Three found a positive association with extremes of precipitation $[11,13,14]$, and one found an inverse association between waterborne outbreaks and average precipitation [12]. Among the two studies that assessed the association with temperature, one found a significant positive association [11]. Of the twenty articles using cases of waterborne infection as study units, amount of precipitation was found to have a positive association with infection in nine of them $[15,16,19,22,24,26,28,29,32]$. Two studies found a positive association in both extremes of precipitation (low and high) [20,27] and six did not find an association $[17,18,21,25,31,34]$. In three studies, statistically significant results were heterogeneous depending on the diseases or geographical regions they were assessing $[23,30,33]$. Regarding temperature, seven studies found a direct association between infections and temperature $[17,18,20,24,25,27,32]$ and four did not find an statistical association $[16,31,33,34]$. In one study, statistically results depended on the disease that was being studied [28].

\section{Discussion}

This review has identified twenty four analytical research studies in which epidemiological and meteorological data have been linked in order to assess associations between extreme precipitation or air temperature and waterborne outbreaks or cases of infection. The findings presented in the different articles are heterogeneous, highlighting the complex relationship between precipitation or temperature driven transmission and waterborne infections. Although 
most of the studies identified a positive association between increased precipitation or temperature and infection, there were several in which this association was not evidenced. A number of articles also identified an association between decreased precipitation and infections. Very few articles presented stratified analyses that took into account the type of water treatment, water source or water supply involved.

Although research on this topic has been performed in different continents, most of the studies were conducted in Asian countries. Only few articles have presented data from Europe or Africa and none presented results from South America, resulting in limited evidence-based information on the influence of extreme weather on waterborne infections in these regions. Most of the publications used cases of infection as study units and only four used outbreaks as units. Of those using cases of infection, cholera or cases of gastroenteritis without a specific etiology were the infections most frequently studied. A variety of study designs and statistical methods, mainly count model regressions and case-crossover analysis, were used.

Several limitations and challenges of the studies were stated by the authors of the reviewed studies. Underreporting is an inherent problem in surveillance systems, and with respect to waterborne outbreaks or infections, the notified cases likely represent just the tip of the iceberg of the true disease burden [35]. However, in terms of estimating the association between weather events and infections or outbreaks, underreporting would only be the cause of bias if reporting is correlated with weather variables [36]. There is lack of consensus about the definition of extreme precipitation or temperature. An association might be found more easily depending on the threshold level that was used to classify extreme precipitation or temperature events. The classification of an extreme weather event is a key issue and needs to be defined according to the regional meteorological pattern. In certain occasions, small data sets in terms of number of observations limit statistical power. One possible solution for sparse data is to aggregate explanatory and outcome variables by week, month or year. However, this may reduce the variation in the data and smooth the relationships with previous weather events. Extreme weather events generally occur on a local scale. This implies that the results obtained from analyzing national, regional or local level will be different and may have noticeable consequences for the interpretations. As an example, presenting results by census area unit instead of national level could allow for variation in exposure across a region or country, although this is not always possible due to limited availability of data. The optimal choice of time lag between weather event and occurrence of a given waterborne disease event is challenging, as these events generally do not occur simultaneously. Using the same time lag for all cases linked to specific weather events is not possible given the variation in incubation periods among and within different infections. Understanding all these issues is necessary in order to select the time lag most relevant for a given disease.

Our review has covered a period of 13 years and has used four different databases, two medical and two multidisciplinary, to identify potential relevant peer reviewed publications in a systematic way. Although relevant literature could have been missed for a number of reasons (not peer reviewed, published before 2001 or in other languages than English, not identified by our search terms, unpublished results), our results show that there is potential to generate more scientific evidence to better understand the association between extreme precipitation or air temperature and waterborne outbreaks or cases of infection.

\section{Conclusion}

The heterogeneity of results presented in the articles identified in this review reflect the complexity of the relationship between extreme precipitation or air temperature and waterborne disease. There are several factors that could play a role on it, such as the specific type of microorganism, the geographical region, season, type of water supply, water source or water treatment. We encourage researchers to conduct studies examining these potential effect modifiers, in order to assess how they modulate the relationship between heavy rain events or temperature and disease. Addressing the gaps will be central for public health experts in order to identify the priority areas where action is needed to minimize negative impact on the health in future climate.

\section{Abbreviations}

WHO: World Health Organization; ECDC: European Centre for Disease Prevention and Control.

\section{Competing interests}

The authors declare that they have no competing interests.

\section{Authors' contributions}

BGH, BFB, KN and LV conceived the study question and the search strategy. JS, BS and GN provided input to the methods proposal and search strategy. $\mathrm{EM}$ and $\mathrm{BGH}$ ran the search strategy and reviewed the titles, abstracts and full texts. BFB reviewed the full texts. All authors participated in manuscript writing and revision. All authors read and approved the final manuscript.

\section{Acknowledgements}

This review has been performed as part of the ECDC commissioned project "Waterborne outbreaks and climate change" (OJ/06/02/2012-PROC/2012/011). We would like to thank Vidar Lund, Preben Ottesen and Wenche Jacobsen from the Norwegian Institute of Public Health for their input on the search strategy; and Margareta Löfdahl from Public Health Agency of Sweden for her input on the manuscript.

\section{Author details}

Department of Infectious Disease Epidemiology, Norwegian Institute of Public Health, Oslo, Norway. ${ }^{2}$ Oslo Centre for Statistics and Epidemiology, Department of Biostatistics, Institute of Basic Medical Sciences, University of Oslo, Oslo, Norway. ${ }^{3}$ European Programme for Intervention Epidemiology Training (EPIET), European Centre for Disease Prevention and Control, Stockholm, Sweden. ${ }^{4}$ European Centre for Disease Prevention and Control, 
Stockholm, Sweden. ${ }^{5}$ Gastrointestinal, Emerging and Zoonotic Diseases Department, Public Health England, London, UK. ${ }^{6}$ Norwich Medical School, University of East Anglia, Norwich, UK. ${ }^{7}$ Department of Hygiene \& Epidemiology, University of Thessaly, Thessaly, Greece.

Received: 20 May 2014 Accepted: 4 March 2015

Published online: 27 March 2015

\section{References}

1. Moors E, Singh T, Siderius C, Balakrishnan S, Mishra A. Climate change and waterborne diarrhoea in northern India: impacts and adaptation strategies. Sci Total Environ. 2013;468-469(Suppl):S139-51.

2. Semenza JC, Menne B. Climate change and infectious diseases in Europe. Lancet Infect Dis. 2009;9:365-75.

3. Schijven J, Bouwknegt M, Husman AM, Rutjes S, Sudre B, Suk JE, et al. A decision support tool to compare waterborne and foodborne infection and/or illness risks associated with climate change. Risk Analysis. 2013;33:2154-67.

4. Semenza JC, Herbst S, Rechenburg A, Suk JE, Hoser C, Schreiber C, et al. Climate change impact assessment of food- and waterborne diseases. Crit Rev Environ Sci Technol. 2012;42:857-90.

5. Semenza JC, Suk JE, Estevez V, Ebi KL, Lindgren E. Mapping climate change vulnerabilities to infectious diseases in Europe. Environ Health Perspect. 2012;120(3):385-92.

6. Cann KF, Thomas DR, Salmon RL, Wyn-Jones AP, Kay D. Extreme water-related weather events and waterborne disease. Epidemiol Infection. 2013;141:671-86.

7. Atlas of Health and Climate. Joint publication World Health Organization and Meteorogical World Organization. 2012. Available at http://www.who. int/globalchange/publications/atlas/en/.

8. European Centre for Disease Control. Climate change. Climate change in Europe. Available at http://www.ecdc.europa.eu/en/healthtopics/climate_ change/Pages/index.aspx.

9. Beniston M, Stoffel M, Harding R, Kernan M, Ludwig R, Moors E, et al. Obstacles to data access for research related to climate and water: implications for science and EU policy-making. Environ Sci Pol. 2012;17:41-8.

10. Semenza JC, Houser C, Herbst S, Rechenburg A, Suk JE, Frechen T, et al. Knowledge mapping for climate change and food- and waterborne diseases. Crit Rev Environ Sci Technol. 2012;42:378-411.

11. Thomas KM, Charron DF, Waltner-Toews D, Schuster C, Maarouf AR, Holt JD. A role of high impact weather events in waterborne disease outbreaks in Canada, 1975-2001. Int J Environ Health Res. 2006;16:167-80.

12. Yang K, LeJeune J, Alsdorf D, Lu B, Shum CK, Liang S. Global distribution of outbreaks of water-associated infectious diseases. PLoS Neglected Tropical Diseases [electronic resource]. 2012;6:e1483.

13. Nichols G, Lane C, Asgari N, Verlander NQ, Charlett A. Rainfall and outbreaks of drinking water related disease and in England and Wales. J Water Health. 2009; $7: 1-8$.

14. Curriero FC, Patz JA, Rose JB, Lele $\mathrm{S}$. The association between extreme precipitation and waterborne disease outbreaks in the United States, 1948-1994. Am J Public Health. 2001;91:1194-9.

15. Eisenberg $M C$, Kujbida G, Tuite $A R$, Fisman DN, Tien JH. Examining rainfall and cholera dynamics in Haiti using statistical and dynamic modeling approaches. Epidemics. 2013;5:197-207.

16. Harper SL, Edge VL, Schuster-Wallace CJ, Berke O, McEwen SA. Weather, water quality and infectious gastrointestinal illness in two Inuit communities in Nunatsiavut, Canada: potential implications for climate change. EcoHealth. 2011:8:93-108.

17. Hu W, Tong S, Mengersen K, Connell D. Weather variability and the incidence of cryptosporidiosis: comparison of time series poisson regression and SARIMA models. Ann Epidemiol. 2007;17:679-88.

18. Louis VR, Gillespie IA, O'Brien SJ, Russek-Cohen E, Pearson AD, Colwell RR. Temperature-driven campylobacter seasonality in England and Wales. Appl Environ Microbiol. 2005;71:85-92.

19. Sasaki S, Suzuki H, Fujino Y, Kimura Y, Cheelo M. Impact of drainage networks on cholera outbreaks in Lusaka, Zambia. Am J Public Health. 2009:99:1982-7.

20. Singh RB, Hales $S$, de Wet N, Raj R, Hearnden M, Weinstein P. The influence of climate variation and change on diarrheal disease in the Pacific Islands. Environ Health Perspect. 2001;109:155-9.

21. Teschke K, Bellack N, Shen H, Atwater J, Chu R, Koehoorn M, et al. Water and sewage systems, socio-demographics, and duration of residence associated with endemic intestinal infectious diseases: a cohort study. BMC Public Health. 2010;10:767.

22. Tornevi A, Axelsson G, Forsberg B. Association between precipitation upstream of a drinking water utility and nurse advice calls relating to acute gastrointestinal illnesses. PLoS ONE [Electronic Resource]. 2013;8:e69918.

23. Vollaard AM, Ali S, Van Asten HAGH, Widjaja S, Visser LG, Surjadi C, et al. Risk factors for typhoid and paratyphoid fever in Jakarta, Indonesia. J Am Med Assoc. 2004;291:2607-15.

24. Wang LX, Li XJ, Fang LQ, Wang DC, Cao WC, Kan B. Association between the incidence of typhoid and paratyphoid fever and meteorological variables in Guizhou, China. Chin Med J. 2012;125:455-60.

25. White AN, Kinlin LM, Johnson C, Spain CV, Ng V, Fisman DN. Environmental determinants of campylobacteriosis risk in Philadelphia from 1994 to 2007. EcoHealth. 2009;6(2):200-8.

26. Drayna P, McLellan SL, Simpson P, Li SH, Gorelick MH. Association between rainfall and pediatric emergency department visits for acute gastrointestinal illness. Environ Health Perspect. 2010;118:1439-43.

27. Hashizume M, Armstrong B, Hajat S, Wagatsuma Y, Faruque AS, Hayashi T, et al. Association between climate variability and hospital visits for non-cholera diarrhoea in Bangladesh: effects and vulnerable groups. Int J Epidemiol. 2007:36:1030-7.

28. Britton E, Hales S, Venugopal K, Baker MG. The impact of climate variability and change on cryptosporidiosis and giardiasis rates in New Zealand. J Water Health. 2010:8:561-71.

29. Chen MJ, Lin CY, Wu YT, Wu PC, Lung SC, Su HJ. Effects of extreme precipitation to the distribution of infectious diseases in Taiwan, 1994-2008. PLoS ONE [Electronic Resource]. 2012;7:e34651.

30. Constantin de Magny G, Murtugudde R, Sapiano MR, Nizam A, Brown CW, Busalacchi AJ, et al. Environmental signatures associated with cholera epidemics. Proc Natl Acad Sci U S A. 2008;105:17676-81.

31. Emch M, Feldacker C, Yunus M, Streatfield PK, DinhThiem V, Canh do G, et al. Local environmental predictors of cholera in Bangladesh and Vietnam. Am J Trop Med Hygiene. 2008;78:823-32.

32. Jutla A, Whitcombe E, Hasan N, Haley B, Akanda A, Huq A, et al. Environmental factors influencing epidemic cholera. Am J Trop Med Hygiene. 2013;89:597-607.

33. Kelly-Hope LA, Alonso WJ, Vu DT, Dang DA, Do GC, Lee H, et al. Geographical distribution and risk factors associated with enteric diseases in Vietnam. Am J Trop Med Hyg. 2007;76:706-12.

34. Rind E, Pearce J. The spatial distribution of campylobacteriosis in New Zealand, 1997-2005. Epidemiol Infection. 2010;138:1359-71.

35. Wheeler JG, Sethi D, Cowden JM, Wall PG, Rodrigues LC, Tompkins DS, et al. Study of infectious intestinal disease in England: rates in the community, presenting to general practice, and reported to national surveillance. The Infectious Intestinal Disease Study Executive. BMJ. 1999;318:1046-50.

36. Environmental European Agency. Climate change, impacts and vulnerability in Europe 2012. AN indicator-based report. EEA report. No 12/2012. ISSN 1725-9177. http://www.eea.europa.eu/publications/climate-impacts-andvulnerability-2012/

\section{Submit your next manuscript to BioMed Central and take full advantage of:}

- Convenient online submission

- Thorough peer review

- No space constraints or color figure charges

- Immediate publication on acceptance

- Inclusion in PubMed, CAS, Scopus and Google Scholar

- Research which is freely available for redistribution 